\title{
Power electronics-based large-scale integration of renewables in power grids
}

Zhe Chen,' Birgitte Bak-Jensen' and Shuju Hu²

'Department of Energy Technology, Aalborg University; 'Institute of Electrical Engineering (IEE), Chinese Academy of Sciences (CAS)

\begin{abstract}
Introduction
In recent years renewable energy (RE)-generating technologies like photovoltaic (PV) cells and wind turbines have increasingly been connected to the electric power grid. According to (IEA 2019), the future electricity demand is expected to increase faster than overall energy demand and to be covered to a large extent by solar PV and wind power. Most wind-power and PV generation will be connected via power electronic (PE) converters, which are critical in converting DC from PV to AC of the grid, as well as being crucial building blocks for variable-speed wind turbines. Furthermore, PE converters can ensure better control and bring many new features to the power system. There has been significant growth in power-electronic interfaced power sources (PEIPSS) with increasing power capacity. This gives rise to several technical challenges with special focus on the capabilities for managing the fast control and operation of the grid in case of failure so as to ensure reliability and security of the total electrical system. As examples, this could be due to low total system inertia, low short-circuit power and limited possibilities for dynamic voltage support (ENTSO-E Guidance 2017). To guarantee grid stability, the grid must always be operated in a balanced condition with power production matching power consumption. This will require more spinning reserves, which can take the form of rapid control of the power-generating units or of load changes to ensure the balance. Furthermore, the system should also create possibilities for harmonic injections to counteract resonances if several distributed energy resources (DER) with their converters are connected along a grid distribution radial (Lu et al. 2016). Consequently, manufacturers of renewable power generators, including PEIPS, will have to ensure that their generators and plants are adapted to the new grid codes.
\end{abstract}

As a consequence of the fluctuating and stochastic nature of RE power generation, it is difficult to guarantee rigorously planned and instantaneous power production and, depending on the market design, to avoid penalties for excessive deviations from the bids the RE operator has submitted before gate-time closure. Therefore, in recent years, investigations have been conducted into the possibility of introducing energy-storage systems to support wind-power and $P V$ generation, whereby different storage options can be considered, that are applicable to both larger and smaller systems. The work by (Heide et al. 2011) examines Europe's storage and balancing needs for a fully renewable electric power system (i.e., 100\% renewable electrical energy on an annual basis) which often has excess instantaneous wind and solar power generation. A method gaining more and more importance for dealing with the RES fluctuations and ensuring a supply/demand balance is to promote active demand response and cross-sector integration (Ponnaganti et al. 2018). However, Djørup et al. (2018) conclude that the current structure of the Danish electricity market cannot financially sustain a system with 100\% renewable energy penetration (mainly from wind), even though the system modelled in that work utilizes a huge amount of cross-sector integration into other energy sectors. From a technical point of view, new grid codes are continuously being introduced by the transmission system operator (TSO) to ensure stability in the grid when integrating more wind or PV power. On the homepage of the Danish TSO Energinet (Energinet.dk 2020), several grid codes are available depending on the type of generation and size, new grid codes also being launched in order to integrate battery storage. These grid codes are based on the EU regulation Network Codes Requirements for Grid Connection of Generators (NC RFG as of 27 April 2019) and the Demand Connection Code (DCC as of 7 September 2019). (Reddy et al. 2017) compare different grid codes before setting up an appropriate wind-power management system for adaptation to the grid codes. (Mohseni and Islam 2012) review the different international grid codes related to wind power and suggest more global ideas and future trends before conducting a review of wind-generator technologies and their power electronic converters, which have to adapt to these codes. Among the issues related to the grid codes are fault ride-through capabilities (FRT) and power-gradient constraints and possibilities for spinning reserve provision controlled by the converters in the power-generating units. More questions about the provision of short-circuit power and concepts of voltage support are raised by (Jia et al. 2018) for adaptation to the grid codes.

To address the above issues in more detail, this chapter will be organized as follows: section 1 will describe the relevant grid codes and the main issues regarding con- 


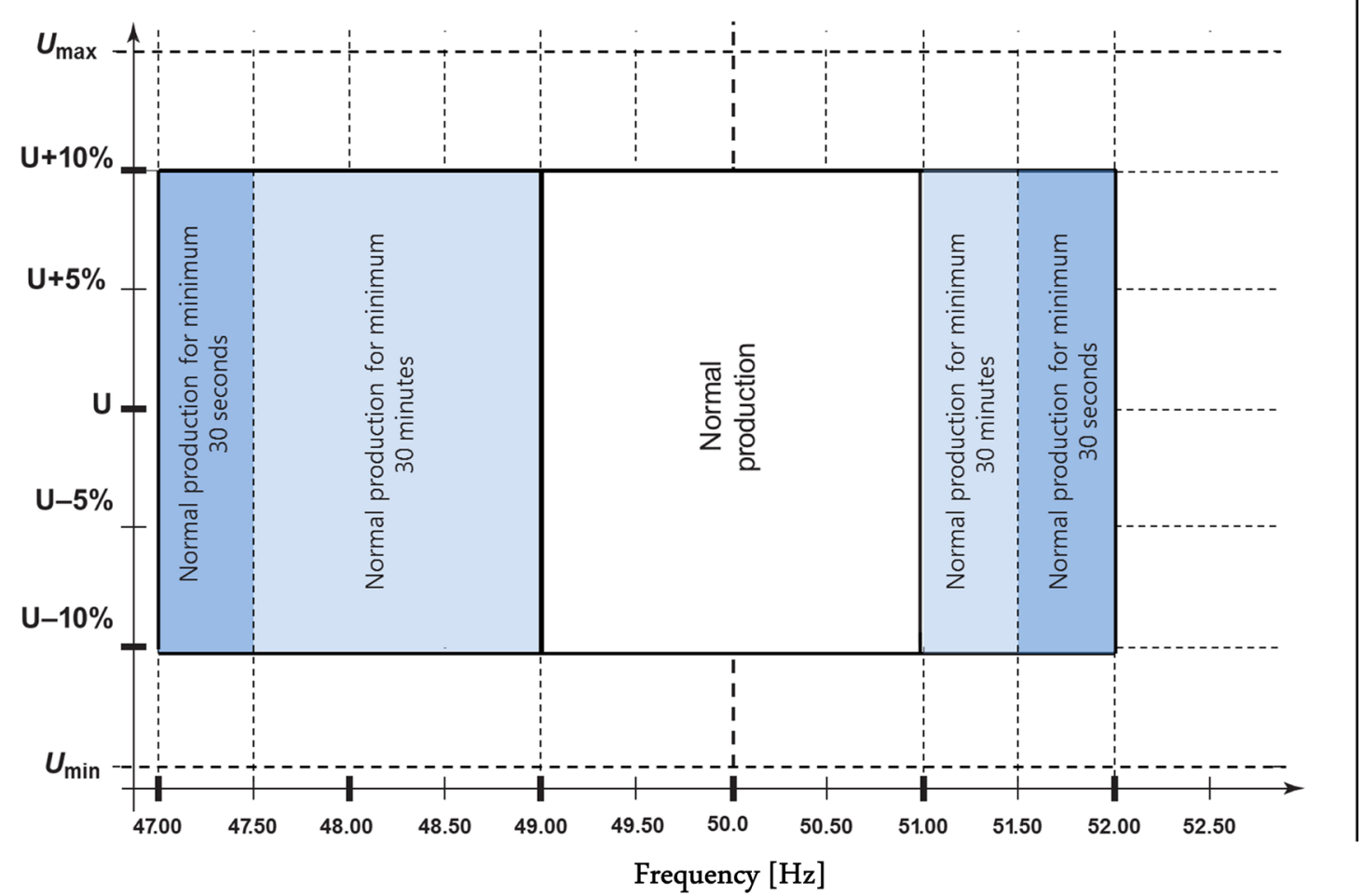

Figure 1 The required frequency/voltage operation range for wind-power plants with nameplate capacity ranging from 25 kW to $1.5 \mathrm{MW}$ (Energinet 2016 ). U and $5 \mathrm{OHz}$ are the 'nominal' values.

trol and safety issues to which the PE interface must adapt. Section 2 will first review the balancing problem, including the power-gradient constraints and voltage-support issues, and then consider the application of storage and demand-response methods and how they can be applied together with the power electronic control of the RE power-generating units. Section 3 will address the problem of power quality by looking at harmonic injections and resonance issues in distribution systems with several parallel connected PE converters. Section 4 will include discussions and recommendations for the application of PE in relation to RE penetration. Finally, section 5 concludes the chapter.

\section{Grid codes for power electronics-interfaced renewable power-generators}

As already noted, large-scale renewable electrical energy (RE) technologies of the naturally fluctuating type, such as wind and PV generators, have steadily increased in installed capacity and will become a very significant part of the generating capacity of many different power systems, which consequently presents huge challenges to power system security. In general, modern RE generators use power electronic converters for connection to the grid and have very different features from the conventional synchronous generators. In order to ensure the stable, reliable and economic operation of power grids with a massive integration of RE power, grid operators must draw up regulations in the form of grid codes to secure reliable operation of the grid with the large-scale penetration of renewable power.
Grid codes for wind and PV generators specify the requirements that they must meet to operate in a power grid. The grid codes cover many different technical aspects usually including steady-state performances (frequency, voltage, active and reactive power, and power quality); dynamic performances (frequency gradients, start-stop, active power ramp rates, reactive power and voltage dynamic control, fault ride-through, inertia, overvoltage and protection); the communication and control interfaces; simulation models; certification and verification; and commissioning and performance verification (Chen 2018). In this section, some main aspects of the Danish grid codes (Energinet 2016) for wind turbines are used as illustrative examples.

The two very important grid operational parameters are frequency and voltage. A power-system operator needs to keep grid frequency and voltage within the specified ranges by assuring that suppliers balance the active and reactive power production with the active and reactive load consumption. With further increases in wind power, conventional coal-fired plants will be taken out of service, leading to reduced dispatchable capacity and a reduced ability to balance grid power as a consequence. Therefore, the grid code requires wind-turbine operators to take greater responsibility for balancing the power, for example, to operate outside the normal conditions for some specified time periods so that the system operator has the time to take the necessary actions to restore normal operations. The 


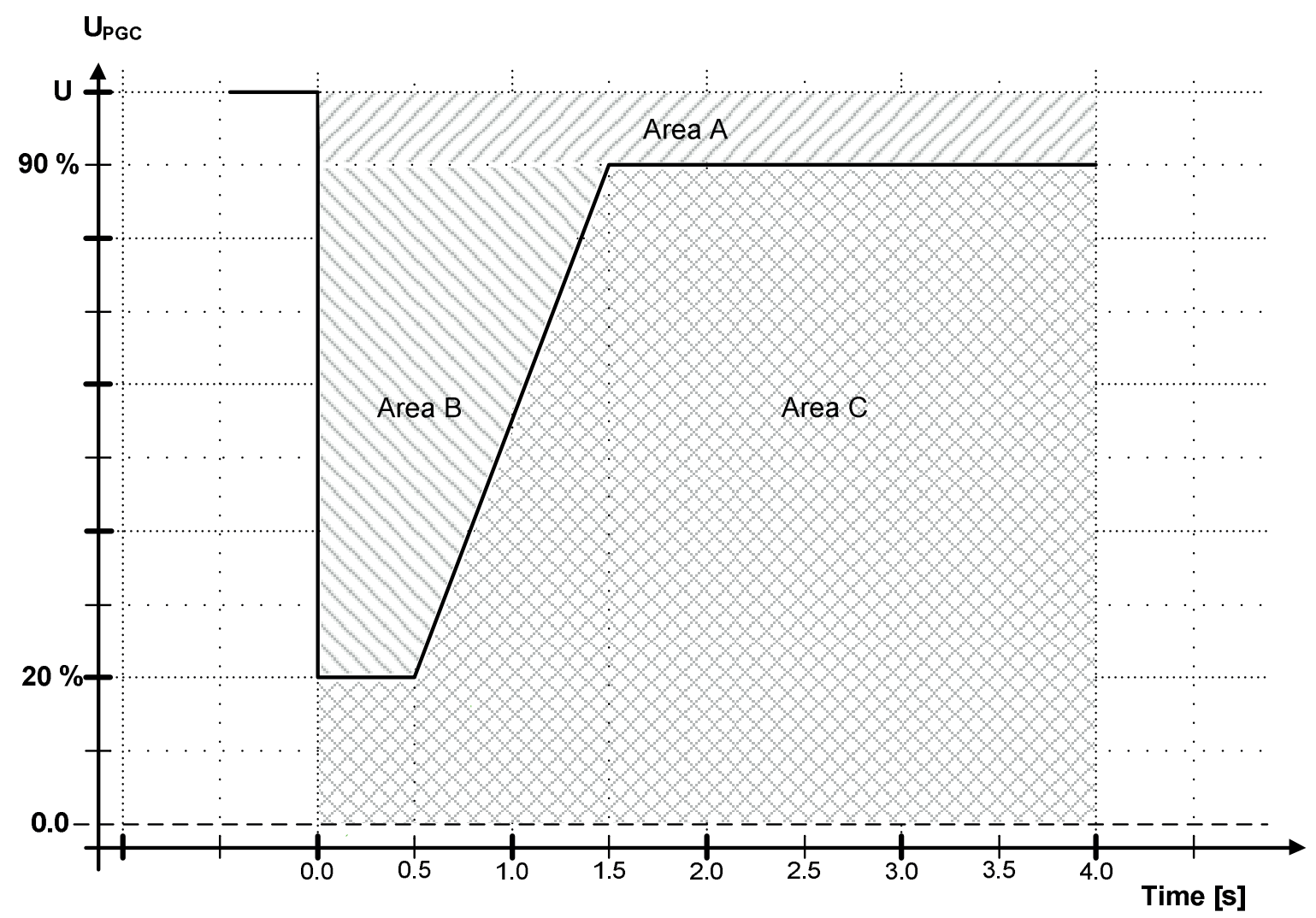

Figure 2 Requirement for tolerance of voltage drops, 'fault ride-through' (for wind turbine with an installed capacity > 1.5 MW) (Energinet 2016).

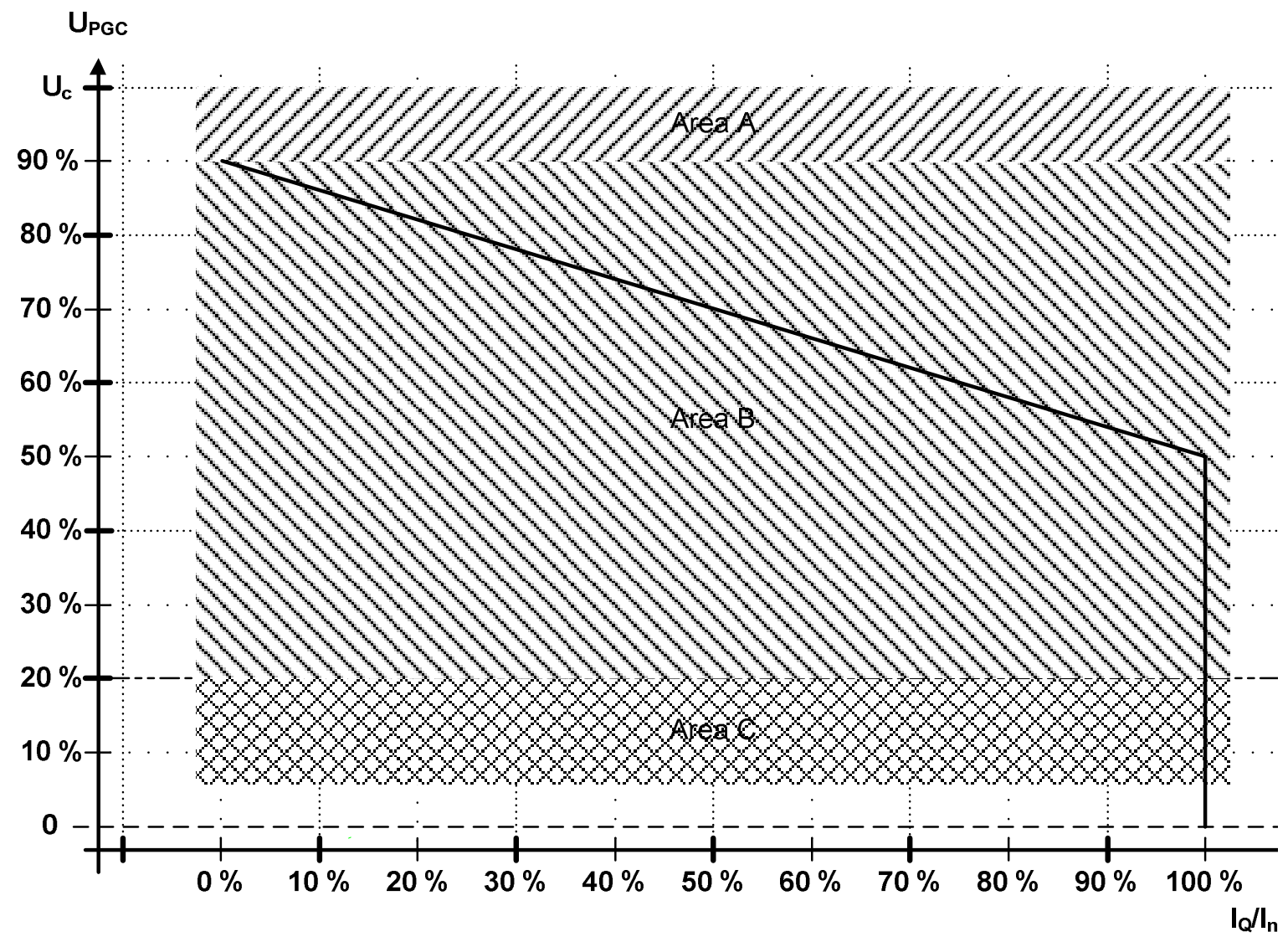


requirements will depend on the installed capacities of the wind-power plants. For example, Figure 1 shows the specified frequency/voltage operation range for wind turbines with nameplate capacity between $25 \mathrm{~kW}$ and $1.5 \mathrm{MW}$ (Energinet 2016).

To contribute to the regulation of frequency, wind turbines are required to adjust their power output to frequency variation. A number of modes of operation should be available at every wind farm and be ready to be put into operation. They may include:

- Balance control: wind farm production can be adjusted up or down to specified levels.

- Delta control: the wind farm is operated with a certain constant reserve power band in relation to its available power production level; such reserves may be used for frequency/power control.

- Power ramp rate limiter: the speed at which the wind farm's power output can be adjusted.

- Reactive power control: the wind farm's reactive power output may be adjusted to a specified level.

- Automatic voltage control: the wind farm may automatically adjust its reactive power production in response to the voltage variation at the measuring point.

In an AC circuit, the product of voltage and current, $\mathrm{VI}$, is expressed as volt-amperes (VA) or kilo volt-amperes (kVA) and is known as Apparent power. For an $A C$ resistive circuit, such as heaters, irons, kettles etc., the current and voltage are in-phase, the dissipated power is real power and equal to the Apparent power. However, if the circuit contains reactive components, the voltage and current waveforms will be "out-ofphase", the Apparent power is not the active power any more. If the angle between the voltage and current is $\Phi$, Reactive Power (sometimes referred to as imaginary power) is expressed by the equation: VIsin $\Phi$ in a unit called "volt-amperes reactive", (VAr), symbol $Q$, while active power, $P$, is VIcos $\Phi$. Reactive power is required by many types of electrical equipment that uses a magnetic field, such as motors, generators and transformers. It is also required to supply the reactive losses on overhead power transmission lines. In a high voltage system reactive power is closely related to system voltage, insufficient supply of reactive power would result in low voltage.

In order to provide the reactive power capability, reactive power compensation equipment, such as Static VAR Compensators (SVCs) and Static VAR Generators (SVGs; static synchronous compensators-STATCOMs), are often installed on wind farms to provide support in both normal and dynamic/transient conditions, while doubly-fed induction generators (DFIGs) and full-scale power electronic-interfaced wind-power generators can contribute to reactive power regulation with their power-electronic interface systems.

To help the power system recover after a fault, grid codes require wind turbines to have a 'fault ridethrough' capability: that is, wind-turbine systems should remain connected to the electricity grid during a grid fault so that they can support the grid's recovery after the fault has been cleared. Otherwise, the disconnection of large numbers of wind turbines from the grid may result in a significant loss in generating capacity, which consequently may cause difficulties in restoring power-system operations after the fault. Figure 2 shows a 'fault ride-through' requirement, where Area A is the normal operational zone and Area $B$ is the fault ride-through zone: that is, wind turbines should not be disconnected from the grid if the voltage is in Area $B$, while the wind turbine may be disconnected if the voltage is in Area C. To help recover system voltage, reactive power support is very important and is also specified in the grid codes, as illustrated in Figure 3, in which areas A, B and C correspond to areas A, B and C in Figure 2.

The grid code may specify other requirements, for example, that wind turbines should withstand more than one independent fault occurring at intervals of a few minutes. Also, wind turbines should provide good power quality and meet the limits of rapid voltage variations, flicker, harmonics and inter-harmonics. Monitoring and communication systems are also recommended.

The requirements of grid codes may vary from one country to another depending on the grid conditions. For instance, a weak or isolated power network would have more tightened fault ride-through requirements (lower voltage and longer duration of ride-through) than a strongly connected power system. The requirements may also change from one type of RE source to another, such as wind turbines and PV generators. However, the categories of the requirements, such as frequency and voltage range, active power regulation, reactive power operation range and operation under fault conditions, are quite similar

\section{The challenge of balancing with plenty of RE power-generating units}

As mentioned in the grid codes, it is desirable for the connected large and medium-sized RE stations to have some ability to help regulate the output of active and reactive power in order to ensure the normal operation of the power system and further play a supporting role under abnormal grid conditions.

Taking wind power as an example, and starting from the power-generation side, industry and academia have launched a series of studies and attempts to reduce or even eliminate the impact of power fluctuations caused by large-scale wind power. Wind-turbine manufacturers such as GE and VESTAS add frequency control 
and active power-control modules to the wind-turbine controller to ensure that the renewable power output responds to changes of grid frequencies.

Further relevant research in academia is being undertaken to tap the potential of renewable power generation to provide frequency support through pre-assessment, controller and control parameter optimization and other methods. Kang et al. (2016) have evaluated the renewable technology support capacity for the design and optimization of a frequency controller at the RE station side. To improve the frequency response performance of RE and ensure its operational stability, Wilchess-Bernal et al. (2016) have studied an optimization method for controller parameters on the basis of an existing controller design, which can be applied to the adaptation of existing controllers. Starting from the wind farm itself, under the mode of maximum power capture of wind energy, an auxiliary power-regulation system is added to the side of wind farm to improve the impact of its wind power on the power grid.

The auxiliary power-regulation system also relies on energy-storage technology. Future advanced energy-storage technologies may include compressed-air energy storage, flywheel energy storage, battery energy storage, superconducting energy storage, super-capacitor energy storage, ice-cold and heat storage, hydrogen storage, P2G and other energy-storage technologies. In terms of physical form, this includes energy-storage equipment that can be used for the peak-shifting and auxiliary services in large power grids, as well as energy-storage modules for homes, buildings and parks. The energy-storage device can store and release energy in time to ensure the continuity and reliability of the power supply.

It is less economic to rely solely on the power-generation side to ensure the safe and stable operation of the system. Therefore, demand-side response (DR) is used to shape the electricity user's consumption behaviour. Demand response means that users respond to the price of electricity or other incentives by changing the way they consume electricity. The most commonly used price mechanisms based on demand-side response include time-of-use price mechanisms, critical peak-load price mechanisms and real-time price mechanisms, all in frequent use in Denmark and other countries. Through demand response, the load demand can be reduced quickly, and, more broadly, in principle in the future, at any moment, the load could be adjusted to achieve peak-load shifting. At least in principle, it is an accommodating way to generate large-scale RE and safely operate offline systems; however, regulatory and price-signal aspects have to be worked out properly to make it really effective.

In China, most of the areas connected to RE, such as wind and photovoltaic, are at the end of the grid. The demand for reactive power from the wind-power plants and the reactive power loss of the transmission lines increase when the installed wind-power capacity and the output power of the wind-power plant are both high. The voltage level and stability margin are reduced due to insufficient reactive power in the grid and the wind farm lacking reasonable reactive dynamic compensation. As a result, it is easy to produce the phenomenon of low voltage in peak-load period and high voltage in low-load period. In order to solve the above problems, reactive voltage control technology has been studied from the perspective of the wind farm. By controlling the power electronic devices of the grid-connected inverter, the wind farm can adjust its reactive power output in accordance with the local grid voltage and provide voltage support to the local grid, thereby increasing the utilization share of the wind power-connected grids.

Given the large-scale integration of wind power, photovoltaic and other renewable technologies, there are still quite a few issues that need to be studied. For example, the topology and control strategy of high-voltage and high-power DC/DC converters suitable for DC collection and transmission; the methods of dealing with the energy curtailments due to RE random fluctuations.

\section{The problem of power quality in distribution systems}

In recent years, increasing attention has been paid to the power-quality issue of grid-connected renewable power generation such as solar energy, wind energy and so on. Most renewable power needs to be converted into standard AC power through power-electronic devices before it can be incorporated into the system; so it is inevitable that harmonics are injected into the power grid, causing harm mainly reflected in: 1) causing additional loss of power lines; 2) affecting the normal operation of various pieces of equipment; 3) causing resonance in the system; and 4) causing automatic devices and relay protection to malfunction, resulting in measurement error.

Reasonable control of harmonics is of great significance not only to the power grid but also to users. Power Quality Harmonics of Public Power Grid (GB/T 14549-93), one of the power quality series standards issued by China in 1993, stipulates the limits of harmonic voltage at all levels (380V-22OkV) of the public power grid. This has been the universal standard so far, as shown in Table 1. The International Electrotechnical Commission (IEC) has also successively published IEC61000 series electromagnetic compatibility standards and IEC 61400 wind-power standards. When RE is connected to the grid, the standard requirements mentioned above should be met to ensure the stable and economical operation of the system.

The methods for solving harmonic pollution can be divided into the following two categories. First starts 


\begin{tabular}{|c|c|c|c|}
\hline \multirow{2}{*}{$\begin{array}{l}\text { Grid nominal voltage } \\
\text { RV }\end{array}$} & \multirow{2}{*}{$\begin{array}{l}\text { Total voltage harmonic } \\
\text { distortion rate (\%) }\end{array}$} & \multicolumn{2}{|c|}{ Each harmonic voltage contains rate (\%) } \\
\hline & & Odd & Even \\
\hline 0.38 & 5.0 & 4.0 & 2.0 \\
\hline 6 & \multirow{2}{*}{4.0} & \multirow{2}{*}{3.2} & \multirow{2}{*}{1.6} \\
\hline 10 & & & \\
\hline 35 & \multirow{2}{*}{3.0} & \multirow{2}{*}{2.4} & \multirow{2}{*}{1.2} \\
\hline 66 & & & \\
\hline 110 & 2.0 & 1.6 & 0.8 \\
\hline
\end{tabular}

from the harmonic source itself by controlling the power electronic converter so that it does not have the ability to produce harmonics or reduces the content of harmonics. The second is compensation control: if harmonics have been generated, compensation devices are installed in the power grid to compensate for the generated harmonics and reactive power.

Research shows that when RE technology is connected to the grid at the same access location, the larger the access capacity, the higher the harmonic distortion level in the distribution network. When the capacities of the connected units are the same, the closer the access location is to the end of the line, the higher the harmonic distortion level of the distribution network, while the maximum harmonic voltage distortion point will be the end node of the line. From the point of view of reducing the harmonic distortion rate, it is not appropriate to connect RE technologies near the end of the line, but rather to the middle node with heavy load.

RE feeds energy into the grid through grid-connected inverters, which are generally controlled using pulsewidth modulation methods. These devices inject current with a large number of high-frequency components into the grid. In order to filter out high-frequency currents, filters can be added between the inverter and the grid. Guo et al. (2O10) have proposed an LCL filter. Compared with the traditional L filter, the LCL filter provides a high-frequency bypass through the capacitor branch, which greatly attenuates the high-frequency current flowing into the grid and is widely used in high-power equipment. In addition, a source-active power filter or a hybrid filter can be added to inject a compensation current (equal in magnitude and directed opposite to the harmonic current) to the grid to suppress the harmonics.

A good control strategy can reduce the adverse impact of RE grid connection. PI control is the typical control strategy, the control algorithm is simple, and it is widely used in engineering. In addition, Proportional Rresonant (PR) control, repetitive control, fuzzy control, vector control and cross-coupling control etc. have also been applied.
Given the further development and deployment of RE technologies, other types of power supply will be connected to the distribution network; the different types of access to the power supply will affect the harmonics of the distribution network, and this needs further study. At the same time, the management of RE grid connections should be strengthened, and RE power installations should be scientifically and rationally planned to improve safe and stable operation of the grid after connection to it.

\section{Discussion of some issues related to a power electronic-dominated electric power grid}

In transmission systems, there are traditional thyristor-based current-type HVDC systems and the newly developed voltage source converter (VSC)-based HVDC systems. The latter are suitable for multi-terminal topologies, have fast power reserve capabilities and do not require a strong $A C$ grid. Power electronics can also enhance the AC system by means of flexible alternating current transmission systems (FACTS), such as STATCOM for reactive power compensation and system voltage support. In distribution systems, power electronics are also used for reactive power compensation, and system voltage and power quality control, including active filters etc., as well as various types of energy-storage interfaces. On the consumer side, there will be more and more power electronic interfaced loads of various types, such as variable speed drives, heat pumps and electrical vehicles. It is clear that a power electronic-based power grid is emerging. Therefore, the conventional modelling and analysis methods, operation and control strategies have to be re-examined.

The stability of connected power electronic converters has also attracted a lot attention, with significant research efforts being made. For example, multi-loopbased active damping has been proposed to enhance stability by introducing an additional control loop, such as a capacitor-current feedback active damping loop. The controller parameters of an active damping loop may be tuned using a passivity-based method of analysis. However, the accurate parameters of the converter 
LCL filter and controller may be difficult to obtain in practice. Thus, various assessment and identification methods of controller and filter parameters have been investigated, such as a grey-box impedance reshaping method, where the parameters of the converter LCL filter, the current control loop and the capacitor current feedback loop are identified by a vector-fitting (VF) algorithm (Zhou et al. 2019). If instability phenomena occur when the VSC is connected to a weak grid, then the parameters of the current control loop and the active damping loop are re-tuned to reshape the output impedance of the VSC, so that the undesired impedance interaction between the VSC and the weak grid can be avoided.

Large-scale power electronic interfaced RE generators that are integrated into the transmission network would change the power system's short-circuit power capacity, which presents a significant challenge to power-system protection systems. For example, a wind-power plant has different short-circuit features than a traditional synchronous power plant, as the short-circuit current will depend on the type of wind-power plant, its configuration and the power electronic design and control procedure. Normally, a power electronic converter would provide a much more reduced short-circuit current than a synchronous generator of the same capacity. This creates problems with some conventional protection relays. For example, distance protection, having been widely used to protect high-voltage transmission lines, is generally based on the principle of measuring the transition impedance, that is, the ratio of voltage to current. However, there are limitations to the application of conventional power frequency distance protection in PE-dominated power systems due to the fault characteristics associated with power electronic converters, such as a lower short-circuit current, frequency deviation of the fault current, high-order harmonics etc. Some efforts have been made to deal with the protection issue, one example being an improved time-domain distance-protection algorithm in which the fault location and transition resistance-related parameters are estimated directly using instantaneous sampling currents and voltages (Ma et al. 2019). The algorithm takes into account the tolerance for the transition impedance and the fast-response requirement to meet the demand for protection of transmission lines.

\section{Summary}

In this chapter, different issues related to the coordination of control strategies for power electronics applied in the network grid have been discussed. The focus has been especially on the large-scale integration of renewables into the grid at both the distribution and transmission levels. First of all, the grid codes for connecting renewables were addressed, with a particular focus on the requirements and limits of frequency and voltage control. Here, it was mainly the Danish grid codes that were used as an example. In the next section, the focus was on the problem of balancing when more renewables with fluctuating and non-predictable power production are integrated into the grid. This creates a need for more balancing reserves, which can also be obtained in the future from demand response of active loads and from the fast-reacting control methods used for the renewable technologies themselves. Therefore, the grid codes will have to be adapted to this situation. Many intelligent systems can be applied, as discussed in section 2. In sections 3 and 4 the focus was on power quality issues and on the power electronic influence on control and protection of the grid. Here the higher harmonic content generated by the power electronics converters applied to renewable technologies and future loads were discussed. Methods of mitigating the harmonics using different kinds of filters and intelligent control methods were addressed. However, as was also pointed out in the different sections, there are still many issues that remain to be solved in the future. One such issue concerns the power electronic interface and its control methods, which must become more intelligent if they are to ensure stability and reliability. SecondIy, grid-balancing and voltage-stability issues at the network grid also have to be considered. This relates to how to achieve balancing with high penetration of renewables for larger areas, and how to ensure stable voltages on the distribution grid with fluctuating and non-predictable power generation from renewables. Even though many ideas and methods are already being simulated and discussed in the global research environment, they still have to be demonstrated in a real environment to prove their feasibility. 


\section{References}

Chen, Z. 2018. 'Wind Power Plant Control'. In: Encyclopedia of Electrical and Electronics Engineering. Wiley.

DCC. 2019. 'Network Code on Requirements for Grid Connection of Generators (NC RFG): must-read for European electricity producers', https://www.emissions-euets.com/ network-codes/network-code-requirements-for-grid-connection-applicable-to-all-generators-nc-rfg

Djørup, Thellufsen, and Sorknæs. 2018. 'The electricity market in a renewable energy system'. Energy, 162.

Energinet. 2016. Technical regulation 3.2.5 for wind power plants with a power output greater than $11 \mathrm{~kW}$

Energinet. 2020. https://en.energinet.dk/Electricity/ Rules-and-Regulations/Regulations-for-grid-connection

ENTSO-E Guidance. 2017. 'document for national implementation for network codes on grid connection', High Penetration of Power Electronic Interfaced Power Sources (HPOPEIPS).

Guo, X. Q. , Wu, W. Y. and Gu, H. R. 2010. 'Modeling and simulation of direct output current control for Icl-interfaced grid-connected inverters with parallel passive damping'. Simulation Modelling Practice and Theory, 18(7): pp. 946956.

Heide, Greiner, Bremen, and Hoffmann. 2011. 'Reduced storage and balancing needs in a fully renewable European power system with excess wind and solar power generation'. Renewable Energy, 36.

Jia, Yang, Nielsen. 2018. 'A Review on Grid-connected Converter Control for Short Circuit Power Provision under Grid Unbalanced Faults', IEEE Transactions on Power Delivery.

IEA. 2019. World Energy Outlook, ISBN 978-92-64-97300-8

Kang, M. , Kim, K. , Muljadi, E. , Park, J. W. and Kang, Y. C. 2016. 'Frequency control support of a doubly-fed induction generator based on the torque limit'. IEEE Transactions on Power Systems, 31(6): pp. 4575-4583.
Lu, Wang, Loh, Blaabjerg. 2016. 'Resonance interaction of multi-parallel grid-connected inverts with LCL-filter', IEEE Transactions of Power Electronics.

Ma, K., Chen, Z., and Bak, C. L. 2019. 'A Novel Parameter Identification-based Distance Protection Algorithm for Transmission Line Connecting to Wind Power Plant'. The 11th IET International Conference on Advances in Power System Control, Operation and Management (APSCOM 2018) Institution of Engineering and Technology.

Mohseni and Islam. 2012. 'Review of international grid codes for wind power integration: diversity, technology and case for global standard', Elsevier Renewable and Sustainable Energy Reviews, 16(6).

NC RfG (2019), 'Network Code on Requirements for Grid Connection of Generators, Commision regulation (EU) 2016/631' - https://www.entsoe.eu/network_codes/rfg/

Ponnaganti, Pillai, and Bak-Jensen. 2018, 'Opportunities and challenges of demand response in active distribution network's, Wiley Interdisciplinary Reviews: Energy and Environment, 7 .

Reddy, Babu, and Sanjeevikumar. 2017. 'A review on grid codes and reactive power management in power grids with WECS' Springer Advances in Smart Grid and Renewable Energy: pp. 525-539.

World energy outlook. 2019. IEA Flagship report, November 2019 .

Wilchess-Bernal F, Chow J H, and Sanchez-Gasca J J. 2016. 'A fundamental study of applying wind turbines for power system frequency control'. IEEE Transactions on Power Systems 31(2): pp. 1496-1505.

Zhou, W., Wang, Y., Cai, P., and Chen, Z. 2019. 'A Gray-Box Impedance Reshaping Method of Grid-Connected Inverter for Resonance Damping', in Proceedings of ICPE 2019 ECCE Asia, Busan, 2660-2667. 